\title{
Exercise under hyperinsulinaemic conditions increases whole-body glucose disposal without affecting muscle glycogen utilisation in type 1 diabetes
}

\author{
K. Chokkalingam • K. Tsintzas $・$ L. Norton • K. Jewell • \\ I. A. Macdonald • P. I. Mansell
}

Received: 28 July 2006 / Accepted: 19 September 2006 / Published online: 21 November 2006

(C) Springer-Verlag 2006

\begin{abstract}
Aims/hypothesis We examined whole-body and muscle metabolism in patients with type 1 diabetes during moderate exercise at differing circulating insulin concentrations.

Methods Eight men (mean \pm SEM age $36.4 \pm 1.5$ years; diabetes duration $11.3 \pm 1.4$ years; BMI $24.6 \pm 0.7 \mathrm{~kg} / \mathrm{m}^{2}$; $\mathrm{HbA}_{1 \mathrm{c}} 7.9 \pm 0.2 \%$ and $V \mathrm{O}_{2}$ peak $44.5 \pm 1.2 \mathrm{ml} \mathrm{kg}^{-1} \mathrm{~min}^{-1}$ ) with type 1 diabetes were studied on two occasions at rest $(2 \mathrm{~h})$ and during $45 \mathrm{~min}$ of cycling at $60 \%$ maximum $\mathrm{VO}_{2}$ with insulin infused at the rate of either 15 (LO study) or 50 (HI) $\mathrm{mU} \mathrm{m}^{-2} \mathrm{~min}^{-1}$ and blood glucose clamped at $8 \mathrm{mmol} / \mathrm{l}$. Indirect calorimetry, insulin-glucose clamps and thigh muscle biopsies were employed to measure wholebody energy and muscle metabolism.

Results Fat oxidation contributed 15 and 23\% to total energy expenditure during exercise in the $\mathrm{HI}$ and LO studies, respectively. The respective carbohydrate $(\mathrm{CHO})$ oxidation rates were $31.7 \pm 2.7$ and $27.8 \pm 1.9 \mathrm{mg} \mathrm{kg}^{-1} \min ^{-1}(p<0.05)$. Exogenous glucose utilisation rate during exercise was substantially greater $(p<0.001)$ in the HI study $(18.4 \pm$ $\left.2.1 \mathrm{mg} \mathrm{kg}^{-1} \mathrm{~min}^{-1}\right)$ than in the LO study $\left(6.9 \pm 1.2 \mathrm{mg} \mathrm{kg}^{-1}\right.$ $\left.\min ^{-1}\right)$. Muscle glycogen content fell by $\sim 40 \%$ during
\end{abstract}

Chokkalingam and Tsintzas are joint first authors.

K. Chokkalingam $\cdot$ P. I. Mansell

Department of Diabetes and Endocrinology,

Queen's Medical Centre,

Nottingham, UK

K. Chokkalingam $\cdot \mathrm{K}$. Tsintzas $(\bowtie) \cdot \mathrm{L}$. Norton $\cdot \mathrm{K}$. Jewell $\cdot$

I. A. Macdonald

Centre for Integrated Systems Biology and Medicine,

School of Biomedical Sciences, University of Nottingham,

Nottingham NG7 2UH, UK

e-mail: kostas.tsintzas@nottingham.ac.uk exercise in both trials. Muscle glycogen utilisation, muscle intermediary metabolism, and phosphorylation of protein kinase B/Akt, glycogen synthase kinase $3 \alpha / \beta$ and extracellular signal-regulated protein kinase 1 and 2 proteins were no different between interventions.

Conclusions/interpretation In patients with type 1 diabetes, exercise under peak therapeutic insulin concentrations increases exogenous glucose utilisation but does not spare muscle glycogen utilisation. A disproportionate increase in exogenous glucose utilisation relative to the increase in $\mathrm{CHO}$ oxidation suggests an increase in glucose flux through non-oxidative pathways.

Keywords Exercise - Glucose disposal ·

Hyperinsulinaemia $\cdot$ Muscle glycogen $\cdot$ Type 1 diabetes

\author{
Abbreviations \\ CHO carbohydrate \\ ERK extracellular signal-regulated protein kinase \\ GSK glycogen synthase kinase \\ PKBAkt protein kinase B
}

\section{Introduction}

Exercise can play an integral role in the management of type 1 diabetes because of its blood glucose-lowering effect [1]. Adjustments to insulin dose and carbohydrate (CHO) intake are generally necessary to reduce the risk of exerciseinduced hypoglycaemia [2], and patients need to be proactive with their diabetes management to accommodate planned exercise [3]. Current recommendations are for 
patients to reduce insulin doses prior to exercise [4], although in practice many patients continue with customary insulin doses and take additional CHO. The average preprandial and peak therapeutic insulin concentrations in patients using a basal bolus insulin regimen are typically $120-180$ and $420-480 \mathrm{pmol} / 1$, respectively $[5,6]$. By contrast, in non-diabetic subjects, circulating insulin normally declines considerably to $30 \mathrm{pmol} / \mathrm{l}$ or less during exercise, and glucagon concentration increases in order to facilitate substrate mobilisation [7-9]. Depending on the exercise timing and its temporal relationship to the last insulin injection, patients with diabetes will therefore generally be carrying out exercise under markedly hyperinsulinaemic conditions, with an increased risk of hypoglycaemia [4].

In healthy volunteers exercising under physiological hyperinsulinaemia $[8,10]$, exercise and insulin interact synergistically to increase glucose uptake. In contrast, patients with type 1 diabetes exercising at $50 \%$ maximum $V \mathrm{O}_{2}$ under basal insulin concentrations of about $60 \mathrm{pmol} / 1$ show reduced glucose uptake and greater reliance on fat oxidation compared with controls [11]. However, when the intensity of exercise was increased to $70 \%$ maximum $\mathrm{VO}_{2}$, these differences were no longer present. In reality, typical everyday therapeutic insulin concentrations are often significantly greater than the concentrations employed in the above study [5] and not all patients with type 1 diabetes are able to carry out such high-intensity exercise. The impact of moderate exercise $\left(60 \%\right.$ maximum $\left.V \mathrm{O}_{2}\right)$ at normal therapeutic hyperinsulinaemic conditions on whole-body and muscle substrate metabolism in patients with type 1 diabetes has not been investigated.

The underlying cellular mechanisms involved in regulating both insulin- and exercise-mediated glucose uptake are only partly understood. The phosphorylated forms of protein kinase $\mathrm{B}(\mathrm{PKB} / \mathrm{Akt})$, glycogen synthase kinase 3 (GSK3) $\alpha$ and $\beta$ isoforms and extracellular signal-regulated protein kinase (ERK 1,2, also known as EPHB2) are intracellular signalling targets potentially involved in the regulation of both insulin- and exercise-mediated glucose uptake $[12,13]$. However, the impact of hyperinsulinaemia and exercise in patients with type 1 diabetes on these insulin-signalling targets is not known.

The aims of this study were to examine the effects of hyperinsulinaemia approximating preprandial and peak therapeutic insulin concentrations during moderate exercise on: (1) whole-body and muscle substrate metabolism in patients with type 1 diabetes; and (2) key insulin-signalling targets involved in the regulation of glucose uptake in muscle in patients with type 1 diabetes. We hypothesised that exercise under peak therapeutic insulin concentrations would increase glucose utilisation and oxidation and reduce reliance on intramuscular glycogen as a metabolic fuel.

\section{Subjects and methods}

Patients and preliminary measurements Eight recreationally active, C-peptide-negative men with type 1 diabetes were recruited. The mean \pm SEM age and BMI were $36.4 \pm$ 1.5 years and $24.6 \pm 0.7 \mathrm{~kg} / \mathrm{m}^{2}$, respectively. The mean duration of diabetes was $11.3 \pm 1.4$ years and they had reasonably well controlled diabetes with a glycated haemoglobin concentration of $7.9 \pm 0.2 \%$. Six patients were on a basal bolus subcutaneous insulin regimen and two were on twice-per-day premixed insulin injections. All patients were screened to exclude micro- and macrovascular complications. The patients' mean $V \mathrm{O}_{2}$ peak was $44.5 \pm 1.2 \mathrm{ml} \mathrm{kg}^{-1}$ $\min ^{-1}$, as determined during a preliminary visit using an incremental cycling test (Excalibur cycle ergometer; Lode, Groningen, the Netherlands). The criteria used for attainment of $V \mathrm{O}_{2}$ peak were as follows: heart rate response $>90 \%$ of predicted maximum heart rate; respiratory exchange ratio $>1.10$; near-maximal rating of perceived exertion (Borg scale $\geq 19$ ); and a drop in pedalling rate greater than $20 \%$. Patients were familiarised with the exercise protocol during pre-study visits to the laboratory, and were informed of all procedures and risks associated with the experimental procedures before they were asked to give informed consent. All procedures used in this study were performed according to the Declaration of Helsinki and approved by the Nottingham NHS Research Ethics Committee.

Experimental design and protocol Studies were carried out in the fasted state on two occasions in random order with a 2 -week interval between visits. Volunteers maintained an isoenergetic diet and monitored their capillary blood glucose closely prior to the study. They were requested to avoid smoking, alcohol and exercise for 3 days prior to each study visit. The subjects omitted their medium-/longacting insulin for $24 \mathrm{~h}$ prior to the study. On the evening prior to the study, subjects consumed a standardised highCHO $(2 \mathrm{~g} / \mathrm{kg})$ meal, injected their usual short-acting soluble insulin and were admitted to the hospital for a low-dose intravenous insulin infusion to maintain euglycaemia overnight. An antegrade cannula was inserted into a cubital fossa vein for insulin infusion. A retrograde cannula was inserted into a dorsal hand vein for blood sampling to measure glucose during the overnight stay and also for drawing arterialised blood samples the following day during the study with the hand placed in a hot-air box $\left(55^{\circ} \mathrm{C}\right)$. At 08.00 hours the following morning the subjects were transferred to the metabolic unit for the study protocol. Resting $V \mathrm{CO}_{2}$ and $V \mathrm{O}_{2}$ were measured for $20 \mathrm{~min}$ using a ventilated hood system (GEM; Nutren Technologies, Manchester, UK) and the values were used for the calculation of resting energy expenditure and substrate 
oxidation rates. Measurements were made while the subjects were lying supine, undisturbed and awake. Intravenous insulin infusions were given at steady-state rates of either 15 or $50 \mathrm{mU} \mathrm{m}^{-2} \mathrm{~min}^{-1}$ (with an appropriate prime) in random order for a total of $3 \mathrm{~h}$. The 15 and $50 \mathrm{mU}$ clamps were designed to approximate typical preprandial (LO) and postprandial (HI) therapeutic insulin concentrations, respectively $[5,6]$. Blood glucose was clamped at $8 \mathrm{mmol} / \mathrm{l}$ to approximate the average $24 \mathrm{~h}$ blood glucose levels observed in reasonably well controlled patients with type 1 diabetes [5]. This also reflects a realistic blood glucose concentration that patients would aim for prior to exercise. Subjects rested in the supine position for the first 120 min of the study and blood samples were collected at regular intervals. A second resting indirect calorimetry measurement was made during the last $20 \mathrm{~min}$ of the resting period. Following this, patients cycled at $60 \%$ of peak $V \mathrm{O}_{2}$ for $45 \mathrm{~min}$ and the hyperinsulinaemic clamp was continued during cycling. Five-minute expired gas samples (Vmax 29; Sensormedics, Yorba Linda, CA, USA) and blood samples were collected every 15 min during cycling. Heart rate and a rating of perceived exertion using a Borg scale [14] were also measured at $15 \mathrm{~min}$ intervals during exercise. A muscle biopsy was obtained from the vastus lateralis under local anaesthetic before and after cycling, as described previously [15] but with suction applied to the end of the biopsy needle to improve tissue sampling. Two different biopsy sites from the same leg were used for muscle sampling on one visit and the contralateral leg during the second visit. The post-exercise biopsy was taken at least $3 \mathrm{~cm}$ distal to the first site to reduce experimental variability [16]. The samples were immediately frozen in liquid nitrogen. Urine samples were collected during the overnight admission and during the study day for glucose and urea measurements. The urine samples were stored at $-20^{\circ} \mathrm{C}$ in $10 \%$ thymol until analysis.

Blood and urine analysis Whole-blood and urine glucose and blood lactate were measured using a Yellow Springs analyser (YSI 2300 Stat Plus-D; Yellow Springs Instruments, Yellow Springs, OH, USA). All serum and plasma samples were stored at $-80^{\circ} \mathrm{C}$ until analysis. Serum insulin and glucagon were measured using commercially available radioimmunoassay kits from Diagnostic Products (Llanberis, Gwynedd, Wales, UK) and plasma NEFA levels were measured using a kit from Wako Chemicals (Neuss, Germany). Whole blood $\beta$-hydroxybutyrate was measured in perchloric acid (10\%)-treated blood samples [17]. Plasma catecholamines were measured using high-performance liquid chromatography with electrochemical detection [18]. Plasma and urine urea were determined using a commercially available enzymatic kinetic method (Randox Laboratories, Crumlin, Antrim, UK).
Muscle metabolite analysis Muscle samples were stored immediately in liquid nitrogen for later analysis. At a later date, $5-10 \mathrm{mg}$ of the muscle biopsy specimen was freezedried and washed with $40 \%$ petroleum ether to remove fat. Muscle powdering, extraction and measurement of metabolites (free glucose, glucose 6-phosphate, lactate, ATP, phosphocreatine and creatine) were carried out as described previously [19]. Acid hydrolysis of small portions of the muscle extract and of the muscle pellet left over after perchloric acid extractions was carried out to measure macro- and proglycogen, respectively. The resulting hydrolysates were used to determine the glucose residues enzymatically [20]. Acetylcarnitine was determined enzymatically using a radioisotope assay [21].

Protein extraction and western blots Total protein extracts were prepared from 10-20 $\mathrm{mg}$ of frozen biopsy tissue. Samples were first homogenised using a Polytron homogeniser for $30 \mathrm{~s}$ on ice in 10 volumes of buffer containing $50 \mathrm{mmol} / 1 \mathrm{HEPES}, 10 \%$ glycerol, $1 \mathrm{mmol} / 1 \mathrm{EDTA}$, $10 \mathrm{mmol} / 1 \mathrm{NaF}, 1 \mathrm{mmol} / 1 \mathrm{Na}_{3} \mathrm{VO}_{4}, 150 \mathrm{mmol} / 1 \mathrm{NaCl}, 1 \%$ Triton X-100 (pH 7.5) and the protease inhibitors 4(2-aminoethyl)benzenesulphonyl fluoride $(10 \mathrm{mg} / \mathrm{ml})$, leupeptin $(0.1 \mathrm{mg} / \mathrm{ml})$ and pepstatin A $(10 \mu \mathrm{g} / \mathrm{ml})$. The homogenates were then centrifuged at $10,000 \times \mathrm{g}$ for $20 \mathrm{~min}$ at $4^{\circ} \mathrm{C}$ and the supernatant fractions stored at $-80^{\circ} \mathrm{C}$ until analysis. Protein concentrations of whole tissue extracts were measured using the BCA method (Pierce, Cramlington, UK).

Proteins were separated using 5-20\% gradient gels and then transferred overnight to Hybond-C nitrocellulose membranes (Amersham Biosciences, Little Chalfont, UK). After blocking with $5 \%$ BSA for $1 \mathrm{~h}$ at room temperature, the membranes were incubated overnight with primary antibodies for phospho-Akt serine ${ }^{473}$, phospho-GSK $3 \alpha / \beta$ serine $^{21} /$ serine $^{9}$ and phospho-ERK1 threonine ${ }^{202}$, phosphoERK2 tyrosine ${ }^{204}$ (Cell Signaling Technology, Danvers, MA, USA), and desmin (Sigma-Aldrich, St Louis, MO, USA) was used as a control. This was followed by incubation for $1 \mathrm{~h}$ at room temperature with secondary antibodies [goat anti-rabbit horseradish peroxidase (HRP) for Akt and GSK3, Amersham Biosciences; goat antimouse HRP for ERK 1/2, DakoCytomation, Denmark]. All immunoreactive proteins were visualised using ECL plus (Amersham Biosciences) and quantified by densitometry using the Gene Tools version 3.0 software (SynGene Division of Synoptics, Cambridge, UK).

Calculations Exogenous glucose utilisation was calculated from the glucose infusion rates achieved during the insulinglucose clamp [22]. Calculations were made at steady state during the resting period of the clamp (90-120 min) and during the entire $45 \mathrm{~min}$ period of the exercise. The glucose 
infusion rate was corrected for glycosuria. Fasting-, insulinand exercise-mediated $\mathrm{CHO}$ and fat oxidation rates were calculated from the $V \mathrm{O}_{2}$ and $V \mathrm{CO}_{2}$ measurements [23]. Substrate oxidation at rest was corrected for protein oxidation using urinary urea concentration and for changes in the blood urea pool during the experiment [24].

Statistics Statistical analysis was carried out using the software package SPSS version 11.5. Two-way ANOVA (treatment and time) was used to compare data from repeated measures. Student's $t$ test (two-tailed) was used to compare paired data within each infusion rate or at corresponding time points between infusion rates where appropriate. A $p$ value less than 0.05 was considered significant. All data are expressed as mean (SEM).

\section{Results}

All eight patients completed the resting part of the experimental protocol and seven completed the exercise protocol on both visits. The eighth subject was unable to complete the exercise protocol on his visit for the HI trial. He managed to cycle for $25 \mathrm{~min}$ and had to stop because of undue exhaustion. Hence, only resting data are presented for this subject. The subjects cycled at a mean workload of $140 \pm 9 \mathrm{~W}$ during both the LO and the HI trials. The mean oxygen consumptions during exercise were $29 \pm 2$ and $29 \pm 1 \mathrm{ml} \mathrm{kg}^{-1} \mathrm{~min}^{-1}$ in the LO and HI trials, respectively, being almost exactly $60 \%$ of the predetermined maximal aerobic power.

Blood metabolites and hormones Blood glucose concentrations at rest were $8.8 \pm 0.4$ and $8.3 \pm 0.3 \mathrm{mmol} / 1$ during the LO and HI trials, respectively. During exercise, glucose concentrations were $8.1 \pm 0.5$ and $8.1 \pm 0.4 \mathrm{mmol} / \mathrm{l}$, respectively. The serum insulin concentrations at rest were $168 \pm$ 18 and $456 \pm 30 \mathrm{pmol} / 1$ during the $\mathrm{LO}$ and $\mathrm{HI}$ trials, respectively (Fig. 1a). During exercise, there were further modest increases $(p<0.05)$, to $210 \pm 18$ and $576 \pm 42 \mathrm{pmol} / \mathrm{l}$, respectively, despite the insulin infusion rates remaining constant. Fasting plasma NEFA concentrations were comparable in the two interventions and were suppressed with the insulin infusions at rest and during exercise (Fig. 2). However, plasma NEFA concentrations were lower in the HI trial $(p<0.01)$. Baseline $\beta$-hydroxybutyrate concentrations were comparable (LO, $0.4 \pm 0.1 \mathrm{mmol} / \mathrm{l} ; \mathrm{HI}, 0.4 \pm 0.1)$ and were suppressed to concentrations below $0.1 \mathrm{mmol} / 1$ during both interventions at rest and during exercise. Blood lactate concentrations increased during insulin infusion, with higher values achieved during the HI clamp at rest (LO, $0.5 \pm 0.1 \mathrm{mmol} / \mathrm{l} ; \mathrm{HI}, 0.7 \pm 0.1 \mathrm{mmol} / 1 ; p<0.05$ ).
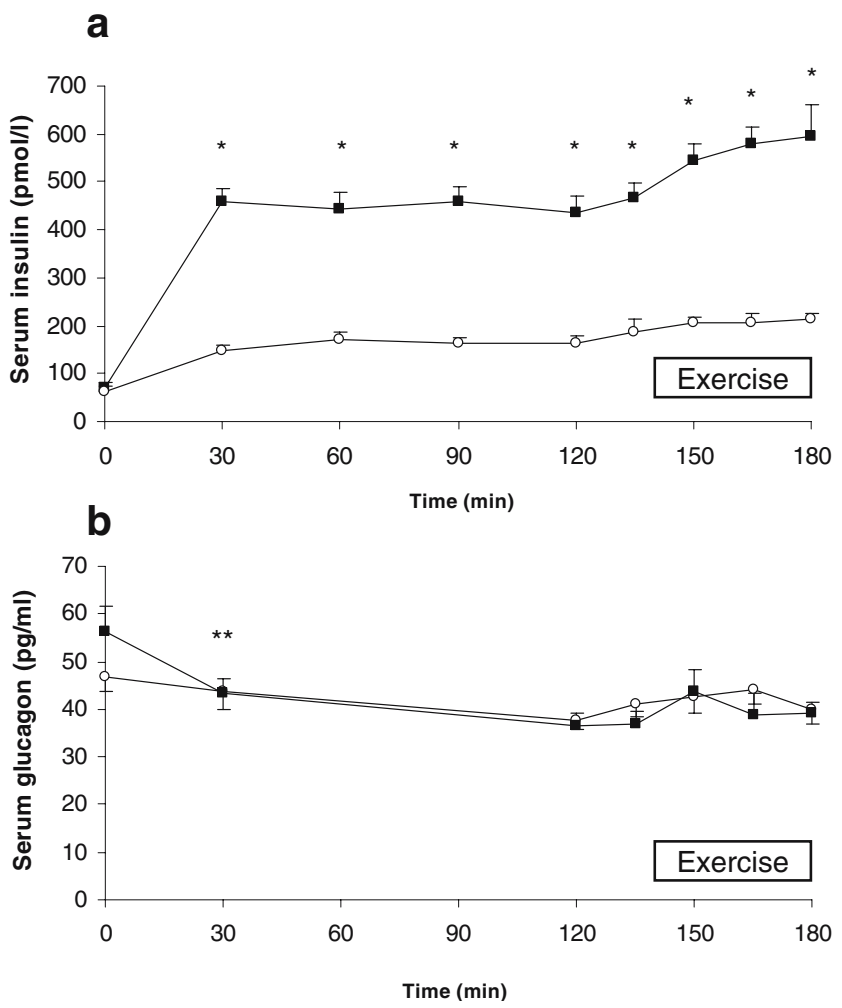

Fig. 1 Serum insulin (a) and glucagon (b) concentrations during the resting period of the clamp and during exercise in the HI (closed squares) and LO (open circles) trials. Data are mean \pm SEM. In panel a, $n=7$ for resting period and $n=6$ for exercise period. ${ }^{*} p<0.001$ for differences in serum insulin concentrations between trials. The value immediately before exercise denotes measurements made in the sitting position. In panel $\mathbf{b}, n=8$ for resting period and $n=7$ for exercise period. ${ }^{* *} p<0.05$ for differences in glucagon concentrations from baseline in the HI trial

There was a further increase during exercise but there was no significant difference between trials (LO, $2.3 \pm 0.5 \mathrm{mmol} / \mathrm{l}$; $\mathrm{HI}, 2.7 \pm 0.6 \mathrm{mmol} / 1$; treatment effect not significant). Serum glucagon concentrations were suppressed by insulin

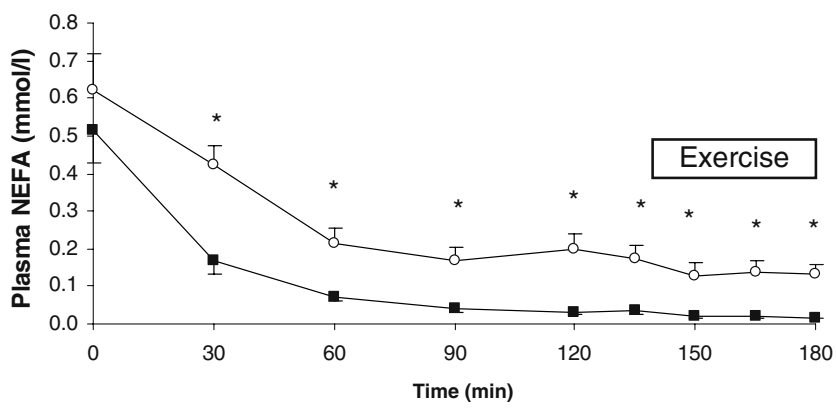

Fig. 2 Plasma NEFA concentrations during the resting part of the clamp and during exercise in the HI (closed squares) and LO (open circles) trials. Data are mean $\pm \mathrm{SEM} ; n=8$ for resting period and $n=7$ for exercise period. The value immediately before exercise denotes measurements made in the sitting position. ${ }^{*} p<0.01$ for differences between trials 


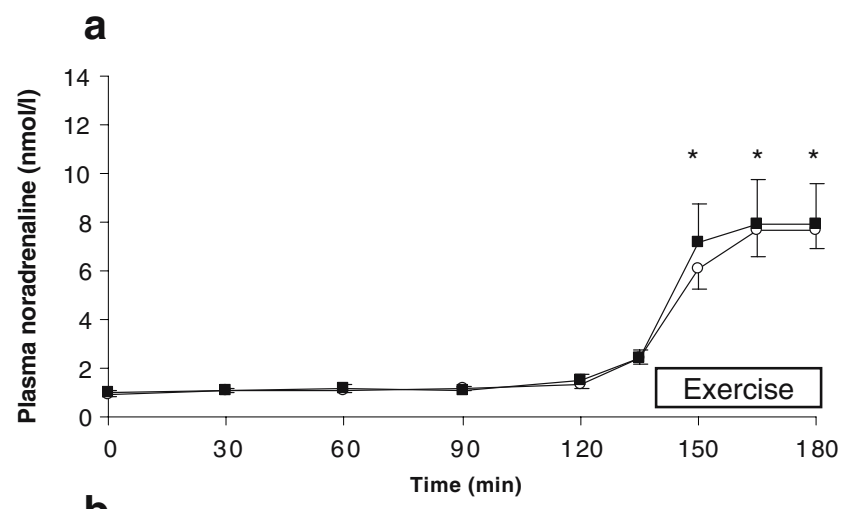

b

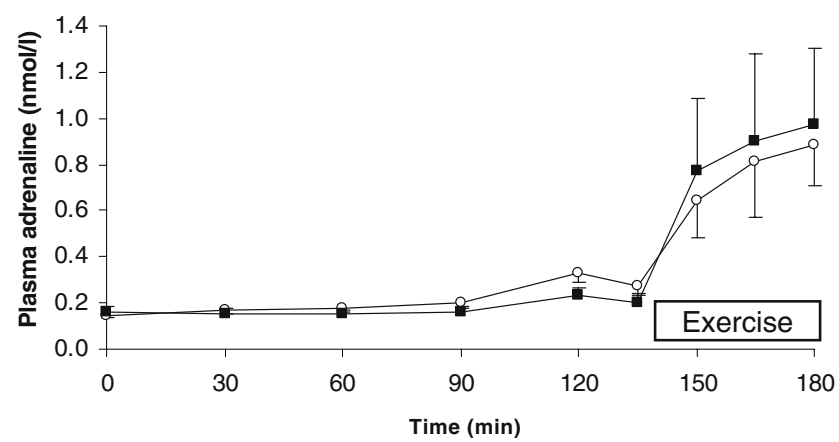

Fig. 3 Plasma noradrenaline (a) and adrenaline concentrations (b) during the resting period of the clamp and during exercise in the $\mathrm{HI}$ (closed squares) and LO (open circles) trials. Data are mean $\pm \mathrm{SEM}$; $n=8$ for resting period and $n=7$ for exercise period. The value immediately before exercise denotes measurements made in the sitting position. There were no differences in catecholamine responses between interventions either at rest or during exercise. ${ }^{*} p<0.05$ indicates that in both trials the concentration of plasma noradrenaline during exercise was significantly higher than during the preceding rest period

infusions after $30 \mathrm{~min}$ in the HI trial $(p<0.05)$ and did not increase during exercise, with no differences between studies (Fig. 1b). There was a marked exercise-induced increase in plasma noradrenaline concentrations during both interventions $(p<0.05)$ but only a non-significant increase in plasma adrenaline was observed. However, there was no difference in catecholamine concentrations between studies at rest or during exercise (Fig. 3a,b).

Whole-body substrate metabolism At rest, insulin-stimulated exogenous glucose utilisation was higher in the HI than in the LO trial $(p<0.001$; Table 1). During both studies, the exogenous glucose utilisation rate increased substantially to maintain concentrations at $\sim 8 \mathrm{mmol} / 1$ when patients changed posture from the supine to the ambulant state. The exogenous glucose utilisation rate increased considerably further $(p<0.001)$ during exercise in both studies but more so in the HI trial (change, $11.3 \pm 1.5 \mathrm{mg} \mathrm{kg}^{-1} \mathrm{~min}^{-1}$ ) compared with the LO trial (difference, $4.6 \pm 1.1 \mathrm{mg} \mathrm{kg}^{-1}$ $\mathrm{min}^{-1}$; Table 1). Exercise also induced a substantial increase in $\mathrm{CHO}$ oxidation during both trials, but it was $15 \%$ greater during the $\mathrm{HI}$ trial than during the LO trial (difference, $3.7 \pm 1.4 \mathrm{mg} \mathrm{kg}^{-1} \mathrm{~min}^{-1} ; p<0.05$; Table 1). During exercise, fat oxidation contributed $\sim 23$ and $15 \%$ of total energy expenditure in the LO and $\mathrm{HI}$ trials, respectively (not significant; Table 1). There was no difference in the mean rating of perceived exertion (Borg scale) during exercise between interventions (LO, 13.3 \pm 0.6 ; HI, 13.1 \pm 0.6 ).

Muscle metabolism Pre-exercise muscle glycogen concentrations were comparable between trials and were reduced by $40-43 \%$ after exercise (Table 2 ). The rate of glycogen utilisation during exercise was similar during the two interventions (LO, 3.9 \pm 0.6 ; HI, $4.1 \pm 0.6 \mathrm{mmol}$ glucosyl units $\mathrm{kg}$ dry muscle ${ }^{-1} \mathrm{~min}^{-1}$ ). There were no differences between interventions in either pro- or macroglycogen utilisation rates. There was no significant increase in intramuscular free glucose concentration during either intervention. There was a three- to fourfold increase in muscle lactate and acetylcarnitine concentrations during

Table 1 Basal and insulin- and exercise-induced substrate oxidation rates, respiratory exchange ratio (RER) and exogenous glucose utilisation rate

\begin{tabular}{|c|c|c|c|c|c|c|}
\hline & \multicolumn{3}{|c|}{ LO trials (low insulin concentration) } & \multicolumn{3}{|c|}{ HI trials (high insulin concentration) } \\
\hline & $\begin{array}{l}\text { Basal } \\
(n=8)\end{array}$ & $\begin{array}{l}\text { Insulin+rest } \\
(n=8)\end{array}$ & $\begin{array}{l}\text { Insulin }+ \text { exercise } \\
(n=7)\end{array}$ & $\begin{array}{l}\text { Basal } \\
(n=8)\end{array}$ & $\begin{array}{l}\text { Insulin+rest } \\
(n=8)\end{array}$ & $\begin{array}{l}\text { Insulin + exercise } \\
(n=7)\end{array}$ \\
\hline Carbohydrate oxidation $\left(\mathrm{mg} \mathrm{kg}^{-1} \min ^{-1}\right)$ & $1.1 \pm 0.4$ & $1.9 \pm 0.3^{\mathrm{a}}$ & $27.8 \pm 1.9$ & $1.4 \pm 0.4$ & $2.7 \pm 0.2^{\mathrm{a}}$ & $31.7 \pm 2.7^{\mathrm{b}}$ \\
\hline Fat oxidation $\left(\mathrm{mg} \mathrm{kg}^{-1} \min ^{-1}\right)$ & $0.8 \pm 0.2$ & $0.5 \pm 0.1$ & $4.1 \pm 0.7$ & $0.8 \pm 0.2$ & $0.1 \pm 0.1$ & $2.5 \pm 0.6$ \\
\hline RER & $0.80 \pm 0.03$ & $0.88 \pm 0.02^{\mathrm{a}}$ & $0.92 \pm 0.01$ & $0.84 \pm 0.04$ & $0.98 \pm 0.02^{\mathrm{a}}$ & $0.95 \pm 0.01^{\mathrm{b}}$ \\
\hline $\begin{array}{l}\text { Exogenous glucose utilisation } \\
\left(\mathrm{mg} \mathrm{kg}^{-1} \mathrm{~min}^{-1}\right)\end{array}$ & 0 & $2.3 \pm 0.4$ & $6.9 \pm 1.2$ & 0 & $7.1 \pm 1.1^{\mathrm{c}}$ & $18.4 \pm 2.1^{\mathrm{c}}$ \\
\hline
\end{tabular}

Data are mean (SEM). Values for insulin+rest are the means for the final $30 \mathrm{~min}$ of the clamp at rest; values for insulin + exercise are the means for the whole $45 \mathrm{~min}$ of the exercise period.

${ }^{a} p<0.05$ compared with the respective baseline

${ }^{\mathrm{b}} p<0.05$ compared with the LO trial

${ }^{c} p<0.001$ compared with the LO trial 
Table 2 Muscle metabolite concentrations before and after exercise

\begin{tabular}{|c|c|c|c|c|}
\hline & \multicolumn{2}{|c|}{ LO trials (low insulin concentration) } & \multicolumn{2}{|c|}{ HI trials (high insulin concentration) } \\
\hline & Before exercise & After exercise & Before exercise & After exercise \\
\hline Total glycogen $(n=7)$ & $406 \pm 21$ & $236 \pm 30^{\mathrm{a}}$ & $403 \pm 22$ & $227 \pm 24^{\mathrm{a}}$ \\
\hline Proglycogen $(n=7)$ & $324 \pm 17$ & $194 \pm 25$ & $323 \pm 20$ & $176 \pm 20$ \\
\hline Macroglycogen $(n=7)$ & $82 \pm 9$ & $42 \pm 6$ & $80 \pm 7$ & $51 \pm 10$ \\
\hline Free glucose $(n=6)$ & $4.4 \pm 0.9$ & $6.9 \pm 1.3$ & $4.2 \pm 2.0$ & $7.6 \pm 2.3$ \\
\hline Glucose 6-phosphate $(n=6)$ & $1.6 \pm 1.0$ & $1.1 \pm 0.2$ & $1.5 \pm 0.3$ & $0.8 \pm 0.2$ \\
\hline Lactate $(n=7)$ & $6.3 \pm 1.5$ & $17.9 \pm 3.9^{\mathrm{a}}$ & $5.6 \pm 1.2$ & $23.8 \pm 3.5^{\mathrm{a}}$ \\
\hline Acetylcarnitine $(n=6)$ & $5.2 \pm 0.3$ & $16.9 \pm 1.0^{\mathrm{a}}$ & $4.2 \pm 0.5$ & $17.0 \pm 2.4^{\mathrm{a}}$ \\
\hline
\end{tabular}

Data are mean \pm SEM millimole glucosyl units per kilogram dry muscle for glycogen and millimole per kilogram dry muscle for metabolites.

${ }^{a}$ Significantly different from the respective baseline $(p<0.05)$

exercise, with no differences between interventions (Table 1). The phosphocreatine/ATP ratios before and after exercise during the $\mathrm{LO}(2.7 \pm 0.1$ and $2.9 \pm 0.2)$ and $\mathrm{HI}$ clamps $(2.9 \pm 0.2$ and $2.8 \pm 0.2$ ) were not different.

Muscle protein phosphorylation Phosphorylations of Akt, GSK3 $\alpha$, GSK $3 \beta$, ERK1 and ERK2 were similar at the end of the $2 \mathrm{~h}$ insulin infusions, with no significant differences between the LO and HI trials. Superimposition of exercise on the hyperinsulinaemic conditions did not alter the phosphorylation of these signalling targets and there were no differences between trials (data not shown).

\section{Discussion}

The principal finding of this study was that exercise under peak therapeutic hyperinsulinaemic conditions in patients with type 1 diabetes did not spare muscle glycogen utilisation at a time of high exogenous glucose utilisation and oxidation. Muscle intermediary metabolism during exercise was not affected by changes in therapeutic insulin concentrations seen routinely in clinical practice (150-540 pmol/1). The insulin-mediated phosphorylation of key signalling targets during exercise did not differ between the two insulin concentrations used.

In patients with type 1 diabetes, exercise under hyperinsulinaemic conditions has a synergistic influence on whole-body glucose utilisation. During exercise, the exogenous glucose infusion rate was substantially increased in both studies to prevent a fall in blood glucose. The amount of glucose infused was substantially higher in the HI trial (68 g) than in the LO trial $(25 \mathrm{~g})$ to maintain blood glucose at $8 \mathrm{mmol} / \mathrm{l}$. Hence, it might have been expected that muscle glycogen would have been spared during the HI trial, but no such differences were observed. Moreover, we did not observe any difference in the concentration of either intramuscular free glucose or glucose 6-phosphate. Intramuscular lactate accumulation (an index of the rate of glycolysis) and acetylcarnitine concentration (an index of pyruvate flux through the pyruvate dehydrogenase complex [25]) were similar during the two trials. Moreover, superimposition of exercise on the hyperinsulinaemic conditions did not influence the phosphorylation of Akt, GSK3 $\alpha$ and $\beta$ and ERK1/2. This is contrary to previously reported findings in healthy volunteers and rats [12, 13, 26, 27]. It is possible that the hyperglycaemic conditions could have had an inhibitory effect on Akt activation, as observed in patients with type 2 diabetes [12]. The above data indicate that at least some of the additional increase in glucose uptake during exercise, especially in the $\mathrm{HI}$ trial, might not have entered contracting muscle. This is in agreement with a previous study which assessed glucose disposal in patients with type 1 diabetes during one-legged isometric exercise under hyperinsulinaemic conditions using 5-fluorodeoxy glucose positron emission tomography [28]. These results suggest the presence of impaired skeletal muscle insulin-stimulated glucose uptake in patients with type 1 diabetes and further investigations are required to confirm this hypothesis.

In the present study, exercise induced a substantial increase in $\mathrm{CHO}$ oxidation in both trials. However, the rate of $\mathrm{CHO}$ oxidation in the $\mathrm{HI}$ trial was only slightly greater $(\sim 15 \%)$ than that in the LO trial. Under fasting conditions, fat oxidation can contribute up to $40 \%$ of energy needs during exercise at moderate intensity in healthy volunteers [29]. However, in the present study the hyperinsulinaemic conditions appear to have impaired the exercise-induced increase in catecholamine-mediated lipolysis [30]. As a result, fat oxidation contributed to less than a quarter of the total energy used to meet the needs of the exercising muscle. Since plasma NEFA availability was suppressed under these conditions, the source of the fat that was oxidised was likely to have been intramuscular triglyceride stores, as seen in other studies [31, 32]. Hence, hyper- 
insulinaemia limits fat utilisation, thereby increasing $\mathrm{CHO}$ oxidation and hence glucose requirements.

Exercising with type 1 diabetes is not straightforward. We observed significant increases in insulin concentrations during exercise in both trials without any change in the insulin infusion rate. This is likely to have been due to reduced clearance. Hence, under real-life conditions exercise not only increases absorption from subcutaneously administered injection depots but also might decrease insulin clearance, leading to high circulating insulin levels during exercise, and increase the risk of exercise-induced hypoglycaemia. Current recommendations are to reduce short-acting insulin doses immediately prior to exercise and to take modest amounts of $\mathrm{CHO}$ during exercise to prevent hypoglycaemia. However, even with reductions in soluble insulin dosages, patients will often be exercising at insulin concentrations similar to those observed in the LO study $(\sim 150 \mathrm{pmol} / \mathrm{l})$, well in excess of the insulin levels seen in non-diabetic subjects during exercise $(\sim 30 \mathrm{pmol} / \mathrm{l})$. Some patients do not reduce insulin doses prior to exercise, but choose instead to take larger amounts of $\mathrm{CHO}$ to forestall hypoglycaemia. Furthermore, patients do not always find it possible to plan meals, insulin doses and exercise rigorously. In practice, exercise is therefore often undertaken at higher postprandial insulin concentrations (similar to the $\mathrm{HI}$ study) requiring additional $\mathrm{CHO}$ intake. The comparative physiology of these two situations has not been described previously in patients with type 1 diabetes.

We have shown here for the first time that, in patients with type 1 diabetes, exercise under differing insulin concentrations is associated with similar energy expenditures and muscle glycogen utilisation rates despite the substantial increase in exogenous glucose utilisation in the HI trial compared with the LO trial. Since it is difficult to estimate precisely the mass of working muscle, it is difficult to quantify the contribution of muscle glycogen to wholebody $\mathrm{CHO}$ oxidation. Nevertheless, the amount of muscle glycogen utilised was similar in the two trials and there was a significant increase in $\mathrm{CHO}$ oxidation on both occasions, with a slightly greater value observed in the HI trial. Interestingly, the increase in exogenous glucose utilisation relative to the increase in $\mathrm{CHO}$ oxidation was much greater in the HI trial compared with the LO trial. This suggests that some of the additional increase in whole-body glucose uptake during exercise in the HI trial might have been directed away from the contracting muscle towards nonoxidative pathways in other tissues. Further studies are required to confirm whether glucose storage occurs in the liver and/or non-contracting muscle tissue during exercise under such conditions. If the site of deposition of excess glucose was indeed the liver, this might potentially reduce the risk of 'late' hypoglycaemia. However, there would be a considerable risk of hypoglycaemia occurring during exercise at high insulin concentrations if $\mathrm{CHO}$ intake were not increased to match energy expenditure.

This study illustrates that even at typical preprandial insulin concentrations (as seen in the LO trial) substrate metabolism during exercise in patients with type 1 diabetes is far from normal, with a low rate of fat oxidation, marked muscle glycogen utilisation and a need for considerable quantities of exogenous glucose to meet energy needs. Exercising at higher, typically postprandial, insulin concentrations increases exogenous glucose utilisation but does not spare muscle glycogen breakdown. In order to further understand the physiological responses to exercise in patients with type 1 diabetes, additional studies are required to investigate the impact of peak insulin concentrations on exercise capacity and muscle fatigue and the role of liver glycogen in maintaining glycaemia during and after exercise.

Acknowledgements We gratefully acknowledge the Special Trustees for Nottingham University Hospital for providing the funding to carry out the study. We would also like to thank M. Bloc, J. Littlewood, S. Sarmad and S. Cordon for their technical assistance during the patient studies and analytical work.

Conflict of interest We declare no conflict of interest with reference to any work carried out in this study.

\section{References}

1. Lawrence RD (1926) The effect of exercise on insulin action in diabetes. Br Med J 1:648-650

2. Kemmer FW (1992) Prevention of hypoglycemia during exercise in type I diabetes. Diabetes Care 15:1732-1735

3. Schiffrin A, Parikh S (1985) Accommodating planned exercise in type I diabetic patients on intensive treatment. Diabetes Care 8:337-342

4. Rabasa-Lhoret R, Bourque J, Ducros F, Chiasson JL (2001) Guidelines for premeal insulin dose reduction for postprandial exercise of different intensities and durations in type 1 diabetic subjects treated intensively with a basal-bolus insulin regimen (ultralente-lispro). Diabetes Care 24:625-630

5. Nielsen FS, Jorgensen LN, Ipsen M, Voldsgaard AI, Parving HH (1995) Long-term comparison of human insulin analogue B10Asp and soluble human insulin in IDDM patients on a basal/bolus insulin regimen. Diabetologia 38:592-598

6. Gulan M, Gottesman IS, Zinman B (1987) Biosynthetic human insulin improves postprandial glucose excursions in type I diabetics. Ann Intern Med 107:506-509

7. Coggan AR, Raguso CA, Gastaldelli A, Williams BD, Wolfe RR (1997) Regulation of glucose production during exercise at $80 \%$ of $\mathrm{VO}_{2 \text { peak }}$ in untrained humans. Am J Physiol 273:E348-E354

8. Wasserman DH, Geer RJ, Rice DE et al (1991) Interaction of exercise and insulin action in humans. Am J Physiol 260:E37-E45

9. Felig P, Wahren J, Hendler R, Ahlborg G (1972) Plasma glucagon levels in exercising man. N Engl J Med 287:184-185

10. DeFronzo RA, Ferrannini E, Sato Y, Felig P, Wahren J (1981) Synergistic interaction between exercise and insulin on peripheral glucose uptake. J Clin Invest 68:1468-1474 
11. Raguso CA, Coggan AR, Gastaldelli A, Sidossis LS, Bastyr EJ 3rd, Wolfe RR (1995) Lipid and carbohydrate metabolism in IDDM during moderate and intense exercise. Diabetes 44:10661074

12. Christ-Roberts CY, Pratipanawatr T, Pratipanawatr W, Berria R, Belfort R, Mandarino LJ (2003) Increased insulin receptor signaling and glycogen synthase activity contribute to the synergistic effect of exercise on insulin action. J Appl Physiol 95:2519-2529

13. Sakamoto K, Arnolds DE, Ekberg I, Thorell A, Goodyear LJ (2004) Exercise regulates Akt and glycogen synthase kinase-3 activities in human skeletal muscle. Biochem Biophys Res Commun 319:419-425

14. Borg GA (1982) Psychophysical bases of perceived exertion. Med Sci Sports Exerc 14:377-381

15. Bergstrom J (1962) Muscle electrolytes in man. Scand J Clin Lab Invest Suppl 68:1-110

16. Costill DL, Pearson DR, Fink WJ (1988) Impaired muscle glycogen storage after muscle biopsy. J Appl Physiol 64:2245-2248

17. Williamson DH, Mellanby J, Krebs HA (1962) Enzymic determination of $\mathrm{D}(-)$-beta-hydroxybutyric acid and acetoacetic acid in blood. Biochem J 82:90-96

18. Forster CD, Macdonald IA (1999) The assay of the catecholamine content of small volumes of human plasma. Biomed Chromatogr 13:209-215

19. Harris RC, Hultman E, Nordesjo LO (1974) Glycogen, glycolytic intermediates and high-energy phosphates determined in biopsy samples of musculus quadriceps femoris of man at rest. Methods and variance of values. Scand J Clin Lab Invest 33:109-120

20. Jansson E (1981) Acid soluble and insoluble glycogen in human skeletal muscle. Acta Physiol Scand 113:337-340

21. Cederblad G, Carlin JI, Constantin-Teodosiu D, Harper P, Hultman E (1990) Radioisotopic assays of CoASH and carnitine and their acetylated forms in human skeletal muscle. Anal Biochem 185:274-278
22. DeFronzo RA, Tobin JD, Andres R (1979) Glucose clamp technique: a method for quantifying insulin secretion and resistance. Am J Physiol 237:E214-E223

23. Frayn KN (1983) Calculation of substrate oxidation rates in vivo from gaseous exchange. J Appl Physiol 55:628-634

24. Jequier E, Acheson K, Schutz Y (1987) Assessment of energy expenditure and fuel utilization in man. Annu Rev Nutr 7:187-208

25. Constantin-Teodosiu D, Carlin JI, Cederblad G, Harris RC, Hultman E (1991) Acetyl group accumulation and pyruvate dehydrogenase activity in human muscle during incremental exercise. Acta Physiol Scand 143:367-372

26. Wojtaszewski JF, Hansen BF, Gade J et al (2000) Insulin signaling and insulin sensitivity after exercise in human skeletal muscle. Diabetes 49:325-331

27. Chen HC, Bandyopadhyay G, Sajan MP et al (2002) Activation of the ERK pathway and atypical protein kinase $C$ isoforms in exerciseand aminoimidazole-4-carboxamide-1-beta-D-riboside (AICAR)stimulated glucose transport. J Biol Chem 277:23554-23562

28. Peltoniemi P, Yki-Jarvinen H, Oikonen V et al (2001) Resistance to exercise-induced increase in glucose uptake during hyperinsulinemia in insulin-resistant skeletal muscle of patients with type 1 diabetes. Diabetes 50:1371-1377

29. Ahlborg G, Felig P, Hagenfeldt L, Hendler R, Wahren J (1974) Substrate turnover during prolonged exercise in man. Splanchnic and leg metabolism of glucose, free fatty acids, and amino acids. J Clin Invest 53:1080-1090

30. Wahrenberg HLF, Engfeldt P, Arner P (1989) Abnormal action of catecholamines on lipolysis in adipocytes of type I diabetic patients treated with insulin. Diabetes 38:524-533

31. Standl E, Lotz N, Dexel T, Janka HU, Kolb HJ (1980) Muscle triglycerides in diabetic subjects. Effect of insulin deficiency and exercise. Diabetologia 18:463-469

32. Wahren JHL (1980) Free fatty acid and ketone body metabolism during exercise in diabetes. Acta Paediatr Scand Suppl 283:39-44 